\title{
Spleen tyrosine kinase facilitates neutrophil activation and worsens long-term neurologic deficits after spinal cord injury
}

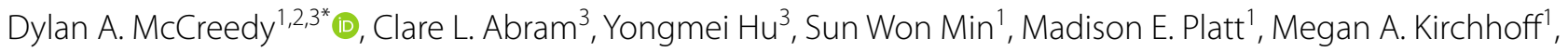
Shelby K. Reid², Frank L. Jalufka' and Clifford A. Lowell ${ }^{3}$

\begin{abstract}
Background: Spinal cord injury elicits widespread inflammation that can exacerbate long-term neurologic deficits. Neutrophils are the most abundant immune cell type to invade the spinal cord in the early acute phase after injury, however, their role in secondary pathogenesis and functional recovery remains unclear. We have previously shown that neutrophil functional responses during inflammation are augmented by spleen tyrosine kinase, Syk, a prominent intracellular signaling enzyme. In this study, we evaluated the contribution of Syk towards neutrophil function and long-term neurologic deficits after spinal cord injury.
\end{abstract}

Methods: Contusive spinal cord injury was performed at thoracic vertebra level 9 in mice with conditional deletion of Syk in neutrophils (Syk $\left.{ }^{f / f} M R P 8-C r e\right)$. Hindlimb locomotor recovery was evaluated using an open-field test for 35 days following spinal cord injury. Long-term white matter sparing was assessed using eriochrome cyanide staining. Blood-spinal cord barrier disruption was evaluated by immunoblotting. Neutrophil infiltration, activation, effector functions, and cell death were determined by flow cytometry. Cytokine and chemokine expression in neutrophils was assessed using a gene array.

Results: Syk deficiency in neutrophils improved long-term functional recovery after spinal cord injury, but did not promote long-term white matter sparing. Neutrophil activation, cytokine expression, and cell death in the acutely injured spinal cord were attenuated by the genetic loss of Syk while neutrophil infiltration and effector functions were not affected. Acute blood-spinal cord barrier disruption was also unaffected by Syk deficiency in neutrophils.

Conclusions: Syk facilitates specific neutrophil functional responses to spinal cord injury including activation, cytokine expression, and cell death. Long-term neurologic deficits are exacerbated by Syk signaling in neutrophils independent of acute blood-spinal cord barrier disruption and long-term white matter sparing. These findings implicate Syk in pathogenic neutrophil activities that worsen long-term functional recovery after spinal cord injury.

Keywords: Spinal cord injury, Neutrophils, Inflammation, Apoptosis, Cytokines, Degranulation, Neutrophil extracellular traps

*Correspondence: dmccreedy@bio.tamu.edu

1 Department of Biology, Texas A\&M University, 301 Old Main Dr, ILSB 3128, College Station, TX 77843, USA

Full list of author information is available at the end of the article

\section{Background}

Spinal cord injury (SCI) results in extensive and persistent inflammation that can exacerbate secondary tissue damage and worsen long-term neurological outcomes. Neutrophils are the most common circulating leukocyte subtype in human blood and the first to invade the 
injured spinal cord in large numbers [1-3]. High abundance of circulating neutrophils early after injury has recently been found to correspond with lower likelihood of functional recovery in individuals with SCI [4-6]. In murine models of SCI, greater neutrophil recruitment from the blood into the acutely injured spinal cord has commonly been associated with increased secondary tissue damage and worsening of neurologic deficits [4, 7-10]. However, antibody-mediated depletion of neutrophils after SCI has produced variable effects on longterm recovery $[4,11-14]$, potentially due to removal of diverse subsets or functions of neutrophils that differentially contribute to tissue damage and repair. The mechanisms by which neutrophils contribute to secondary pathogenesis and neurologic deficits after SCI currently remain unclear.

Neutrophils have a diverse array of cellular responses that enable context-dependent activation of pro- and anti-inflammatory functions $[1,15]$. Activated neutrophils can trigger a variety of cytotoxic effector functions including the production of reactive oxygen and nitrogen species (ROS/RNS), formation of neutrophil extracellular traps (NETs) and degranulation [16-21]. While imperative for sterilizing sites of injury, neutrophil effector functions can also result in collateral damage to adjacent tissue and amplify pathogenic inflammatory responses. Activated neutrophils can also undergo apoptosis at the injury or infection site and are normally phagocytosed and cleared by macrophages. The phagocytosis of apoptotic neutrophils by macrophages has been shown to induce an anti-inflammatory phenotype in the macrophages, which may promote tissue repair and resolution of inflammation [22-25]. However, this process may be impaired in the injured spinal cord where macrophages extensively phagocytose myelin debris and lose their capacity to clear dead neutrophils [26, 27]. The cytotoxic cellular contents of dead neutrophils may then leak in the tissue parenchyma and cause unintended damage. In other models of inflammation, neutrophils have also been shown to produce cytokines that influence inflammation and wound healing [28-31]. Little is known, however, about the diverse functions utilized by neutrophils in the injured spinal cord or the signaling pathways that mediate pathogenic neutrophil responses to SCI.

To enable rapid and specific responses to inflammatory stimuli present at the site of injury or infection, neutrophils express a wide repertoire of immunoreceptors including FcyRs and integrins [32-34]. Spleen tyrosine kinase (Syk) is a prominent signaling mediator in neutrophils that interacts with immunoreceptor tyrosine activation motifs (ITAMs) to help coordinate intracellular responses to the collective stimuli encountered during inflammation [33, 35-37]. Syk can contribute to the development of neutrophil effector functions, as well as cytokine secretion, to facilitate the clearance of microbial infection [38, 39]. While beneficial for fighting pathogenic microbes, Syk has also been found to exacerbate destructive neutrophil responses and tissue damage in multiple inflammatory conditions including arthritis, thrombohemorrhagic vasculitis, epidermolysis bullosa acquisita, and asthma [40-45]. Syk signaling, therefore, may play a critical role in neutrophil responses and functional recovery after SCI.

To determine if Syk contributes to pathogenic neutrophil activities in the injured spinal cord, as well as long-term neurologic deficits, we examined a neutrophilspecific knockout of Syk in a murine thoracic contusion model of SCI. Mice expressing Cre recombinase under control of the MRP8 gene regulator elements (MRP8Cre) were bred with $\mathrm{Syk}^{\mathrm{f} / \mathrm{f}}$ mice to produce conditional deletion of Syk in neutrophils. Following SCI, neutrophil-specific deletion of Syk led to improved long-term functional recovery, but did not reduce long-term white matter tissue loss or acute blood-spinal cord barrier disruption. Syk deficiency attenuated neutrophil activation, cytokine expression, and cell death, but did not alter neutrophil accumulation or effector functions acutely after SCI. Collectively, these findings demonstrates that Syk signaling influences neutrophil phenotype and function in the acutely injured spinal cord and exacerbates longterm neurologic deficits after SCI.

\section{Methods \\ $\mathrm{Syk}^{f / f}$ MRP8Cre mice}

All studies were performed in accordance with protocols approved by the Institutional Animal Care and Use Committee at the University of California, San Francisco. $\mathrm{Syk}^{\mathrm{f} / \mathrm{f}}$ and MRP8-Cre (The Jackson Laboratory) mice were independently maintained on a $\mathrm{C} 57 \mathrm{Bl} / 6$ background and bred to generate $\mathrm{Syk}^{\mathrm{f} / \mathrm{f}}$ and $\mathrm{Syk}^{\mathrm{f} / \mathrm{f}} \mathrm{MRP}$-Cre littermate mice for this study, as previously described $[38,40]$. Adult male and female mice ( $\sim 3$ to 5 months old) were used in all experiments. Mice were housed in groups of two to five prior to injury and female $(\mathrm{F})$ mice were housed in groups of two to three after SCI, whereas males (M) were singly housed.

\section{Spinal cord injury}

Spinal cord contusion injury was performed as previously described [46]. Briefly, mice were anesthetized with $2 \%$ isoflurane, a laminectomy was performed at thoracic vertebrate level 9 (T9), and a $2 \mathrm{~g}$ weight was dropped $7.5 \mathrm{~cm}$ onto the exposed dura mater to produce a moderate contusion SCI [47]. Following the SCI, the cut musculature was sutured together, and the skin incision was closed with wound clips. Mice also received a topical 
administration of bupivacaine at the incision site and a subcutaneous injection of saline. A circulating water pad set at $37{ }^{\circ} \mathrm{C}$ was used to maintain body temperature during and after surgery. For 10 days following SCI, mice received daily subcutaneous injections of saline and antibiotic (enrofloxacin, $2.5 \mathrm{mg} / \mathrm{kg}$ ). Manual bladder expression was performed twice daily until euthanasia.

\section{Experimental design}

The primary goal of this study was to determine the contribution of Syk signaling on neutrophil phenotype and effector functions in the acutely injured spinal cord, as well as long-term functional recovery after SCI. We utilized MRP8-Cre and $\mathrm{Syk}^{\mathrm{f} / \mathrm{f}}$ mice to obtain conditional deletion of Syk in neutrophils as previously described [38, 40]. Syk ${ }^{\mathrm{f} / \mathrm{f}}$ MRP8-Cre mice lack Syk kinase in neutrophils but continue to express normal levels of Syk in other myeloid and B-cell leukocyte subsets. The deficiency of Syk kinase in neutrophils does not alter granulopoiesis; Syk ${ }^{\mathrm{f} / \mathrm{f}} \mathrm{MRP8}$-Cre mice have normal neutrophil numbers in the peripheral blood, spleen and bone marrow. Male and female littermate adult mice were used throughout the study. Sex-specific effects were evaluated for experiments with a sample size of three or more for each sex and in which a significant difference was detected between genotypes. Time points for data collection and sample sizes were determined based on previous studies and experience, as well as power analysis. SCI surgeries and behavioral analysis were performed with the researchers blinded to the genotype. Block randomization was used for all experiments.

\section{Open field locomotor testing}

To assess functional recovery, open field locomotor testing using the Basso Mouse Scale (BMS) was performed at 1,3 , and 7 days post-SCI and then weekly thereafter until euthanasia at five weeks post-SCI. The BMS test assesses multiple aspects of hindlimb movement, paw placement, stepping and coordination [48]. Mice were acclimated to an open field arena $(53 \times 108 \times 5.5 \mathrm{~cm})$ in 4 min sessions on 3 consecutive days during the week prior to injury. After SCI, mice were placed into the open field and scored using the 9-point BMS during a 4-min testing period by observers blinded to the genotype.

\section{White matter sparing}

Animals were euthanized at 35 days post-SCI and transcardially perfused with $35 \mathrm{~mL}$ of PBS followed by $35 \mathrm{~mL}$ of $4 \%$ paraformaldehyde in PBS. The spinal cords $(4 \mathrm{~mm}$ centered over the injury site) were dissected and postfixed overnight at $4{ }^{\circ} \mathrm{C}$. Spinal cords were then cryoprotected in $30 \%$ sucrose, embedded, and frozen at $-80{ }^{\circ} \mathrm{C}$. $20 \mu \mathrm{m}$ transverse cryosections spanning the lesion site were obtained and every tenth section was selected for eriochrome cyanine staining. Images were captured using a Leica DM $6 \mathrm{~B}$ microscope equipped with a $\times 10$ objective (0.32 NA) and a Leica DM45000 color camera. The total area of residual myelin with was hand-traced and quantified using ImageJ. The section with the least total myelin area was selected as the lesion epicenter.

\section{Neutrophil counting}

Animals were euthanized at $1 \mathrm{~d}$ post-SCI and $25 \mu \mathrm{m}$ transverse cryosections were obtained as described above for "White matter sparing". Every twentieth section $(\sim 500 \mu \mathrm{m}$ between sections) was labeled with AlexaFluor647-Ly6G (Clone 1A8, 1:200 dilution, Biolegend), anti-NeuN (1:1000, Millipore), and FluoroMyelin Green (1:300, ThermoFisher Scientific). Sections were then washed and incubated with an AlexaFluor 555-conjugated secondary antibody (1:500, Life Technologies). Images were captured using a Nikon Eclipse upright microscope equipped with a $\times 10$ objective $(0.45 \mathrm{NA})$. Neutrophils were counted using custom Matlab code with regions of interest (ROIs) manually drawn for spinal cord grey matter, white matter, and meninges for each section. The section with the greatest number of neutrophils was designated as the lesion epicenter.

\section{Flow cytometry analysis}

Mice were anesthetized by i.p. injection of $2.5 \%$ avertin ( $0.02 \mathrm{~mL} / \mathrm{g}$ body weight; Sigma) and peripheral blood was obtained by cardiac puncture using a heparin-coated syringe. Approximately $5 \mathrm{~mm}$ of spinal cord tissue centered on the injury site was rapidly dissected and placed into RPMI media on ice. The spinal cord tissue was mechanically dissociated using a plastic tissue pestle and filtered through a $100 \mu \mathrm{m}$ nylon mesh cell strainer. RBC lysis (Pharm Lyse, BD) was performed in all blood and spinal cord samples for $5 \mathrm{~min}$ on ice and quenched with FACS buffer $(0.5 \mu \mathrm{M}$ EDTA and $2 \%$ heat-inactivated fetal bovine serum in HBSS). Following centrifugation, the cell pellet was washed with HBSS and live-dead cell staining was performed using Zombie Dye Red (1:500 dilution, Biolegend) for $30 \mathrm{~min}$ at $4{ }^{\circ} \mathrm{C}$. Samples were washed with FACS buffer and Fc blocking was performed using an anti-mouse CD16/32 antibody (1:100 dilution, Clone 93, eBioscience) for $20 \mathrm{~min}$ at $4{ }^{\circ} \mathrm{C}$. Samples were washed with FACS buffer and partitioned for surface and intracellular staining.

For surface staining, samples were incubated with a cocktail of the following antibodies in FACS buffer for $30 \mathrm{~min}$ at $4{ }^{\circ} \mathrm{C}$ : APC/Cy7-CD45 (Clone 30-F11, 1:200 dilution, Biolegend), APC-CD11b (Clone M1/70, 1:200 dilution, Biolegend), Pacific Blue-Ly6G (Clone 1A8, 1:200 dilution, Biolegend), PerCP/Cy5.5-Ly6C (Clone HK1.4, 
1:600 dilution, Biolegend), and FITC-CD62L (Clone Mel14, 1:100 dilution, Biolegend). Following incubation, samples were washed twice with FACS buffer and counting beads (AccuCheck, ThermoFisher Scientific) were added to the spinal cord samples to determine total cell number.

For ROS labeling, dissociated spinal cord and heparinized blood samples were incubated with DHR123 (1:5000, ThermoFisher Scientific) for $30 \mathrm{~min}$ at $37^{\circ} \mathrm{C}$. The cells were then washed with cold RPMI media and centrifuged at $400 \mathrm{~g}$ for $5 \mathrm{~min}$ at $4{ }^{\circ} \mathrm{C}$. RBC lysis and Fc blocking was performed as described above and samples were incubated with a cocktail of the following antibodies in FACS buffer for $30 \mathrm{~min}$ at $4{ }^{\circ} \mathrm{C}$ : APC/Cy7-CD45, APCCD11b, Pacific Blue-Ly6G and PerCP/Cy5.5-Ly6C.

For intracellular staining, samples were first incubated with the following cocktail of antibodies in FACS buffer for 30 min at $4{ }^{\circ} \mathrm{C}$ : APC/Cy7-CD11b, Pacific Blue-Ly6G, and PerCP/Cy5.5-Ly6C. Samples were then washed twice with FACS buffer and fixed using the Cytofix/Cytoperm kit (BD) according to the manufacturer's protocol. After fixation, samples were washed twice with permeabilization buffer and incubated with one of the following primary antibodies in permeabilization buffer for $30 \mathrm{~min}$ at $4{ }^{\circ} \mathrm{C}$ : AlexaFluor 647-conjugated anti-Syk (1:500, UCSF mAb Core), anti-MRP8 (1:2000, R\&D Systems), or antiCitH3 (1:2000, Abcam). Samples were washed twice with NDS-FACS buffer (HBSS containing 2\% normal donkey serum (NDS) and $0.5 \mu \mathrm{M}$ EDTA) and incubated with an AlexaFluor 488-conjugated secondary antibody (1:500, Life Technologies) in NDS-FACS buffer for $30 \mathrm{~min}$ at $4{ }^{\circ} \mathrm{C}$. Samples were washed twice with NDS-FACS buffer and resuspended for flow cytometry analysis.

Flow cytometry was performed using a Fortessa flow cytometer (BD). Negative and positive fluorescent signals for compensation were obtained using the anti-rat/ hamster and amine reactive compensation bead kits (ThermoFisher Scientific). Flow cytometry data was analyzed and geometric mean fluorescent intensity (G-MFI) values were calculated using FlowJo software (BD). Doublet cells were removed using FSC-H by FSC-A gating. Isotype control (for antibody labeling) or negative staining (for ROS labeling) groups were used to establish gates and to determine background G-MFI values that were subtracted from the reported G-FMI values.

\section{Immunoblotting}

Spinal cord tissue (5 $\mathrm{mm}$ centered over the injury site) was rapidly dissected and homogenized in RIPA buffer (ThermoFisher Scientific) supplemented with a protease inhibitor cocktail (Roche) on ice. Samples were centrifuged to remove insoluble debris and protein concentration in the homogenates was determined using a BCA protein assay kit (Pierce). For each sample, $20 \mu \mathrm{g}$ of protein was loaded into a 4-12\% Bis-Tris gel (ThermoFisher Scientific) and transferred onto a PVDF membrane. The membrane was blocked with $5 \%$ bovine serum albumin (BSA) in Tris-buffered saline (TBS) for $2 \mathrm{~h}$ and then incubated for $1 \mathrm{~h}$ at $4{ }^{\circ} \mathrm{C}$ with donkey anti-mouse IgG IRDye 800 (1:1000 dilution, LI-COR) in TBS containing $0.1 \%$ triton (TBST) and 5\% BSA. The membrane was washed three times with TBST and imaged using an Odyssey CLx imager (LI-COR). GAPDH (Millipore) was used as an internal loading control. The bands at $\sim 50 \mathrm{kDa}$ and $\sim 25 \mathrm{kDa}$ corresponding to the IgG heavy and light chain subunits, respectively, were quantified and normalized to GAPDH using Image Studio Lite software (LI-COR).

\section{Gene expression array}

Neutrophils were isolated from the blood and spinal cords (5 mm centered over the injury site) of Syk ${ }^{\mathrm{f} / \mathrm{f}}$ and $\mathrm{Syk}^{\mathrm{f} / \mathrm{f}} \mathrm{MRP} 8-C r e$ mice at 1 days post-SCI using magnetic bead separation. Neutrophils from the blood from Syk ${ }^{\mathrm{f} / \mathrm{f}}$ uninjured mice were also isolated as a control population. Blood and spinal cord samples were collected and dissociated as described above for "Flow cytometry analysis". Following RBC lysis and Fc blocking, cells were incubated with PE-Ly6G antibody (Clone 1A8, 1:100 dilution, Biolegend) for $20 \mathrm{~min}$ at $4{ }^{\circ} \mathrm{C}$. Cells were washed and then incubated with magnetic UltraPure Anti-PE MicroBeads (1:5 dilution, Miltenyi) for $20 \mathrm{~min}$ at $4{ }^{\circ} \mathrm{C}$. Cells were washed again and labeled neutrophils were isolated using LS columns (Miltenyi) and a QuadroMACS Separator (Miltenyi) according to the manufacturer's protocol. Magnetic isolation was then performed again to further increase the purity of the isolated neutrophil population. All buffers and reagents were kept on ice or at $4{ }^{\circ} \mathrm{C}$ during the isolation to reduce transcriptional changes in the isolated cell population.

Isolated neutrophils from the blood samples were counted and $\sim 2 \times 10^{4}$ purified neutrophils from the blood and $4 / 5$ of the purified neutrophils from the spinal cord were lysed in TRI Reagent (Zymo). The remaining cells were used for flow cytometry analysis to determine neutrophil purity. RNA purification was performed using a Direct-zol RNA Microprep kit (Zymo) according to manufacterur's instructions. Reverse transcription of RNA into cDNA was performed using SuperScript IV VILO (ThermoFisher Scientific). The resulting cDNA was stored at $-80^{\circ} \mathrm{C}$ until further use.

Gene expression analysis of 40 genes was performed using the Biomark 48.48 Dynamic Array (Fluidigm) according to the manufacturer's protocol. Primers (Table 1, IDT or RealTimePrimers.com) were previously validated for specificity using the NIH BLAST search and tested for linearity using serially diluted cDNA from 
Table 1 Primer sequences for gene array

\begin{tabular}{|c|c|c|c|}
\hline Gene symbol & Forward primer $\left(5^{\prime}-3^{\prime}\right)$ & Reverse primer $\left(5^{\prime}-3^{\prime}\right)$ & Accession number \\
\hline Actb & AGGGAAATCGTGCGTGACAT & GAACCGCTCGTTGCCAATAG & NM_007393.5 \\
\hline Angpt1 & GGGGGAGGTTGGACAGTAA & CATCAGCTCAATCCTCAGC & NM_009640.4 \\
\hline $\operatorname{Arg} 1$ & CTCCAAGCCAAAGTCCTTAGAG & AGGAGCTGTCATTAGGGACATC & NM_007482.3 \\
\hline $\mathrm{Bc} \mid 2 \mathrm{a} 1$ & GATACGGCAGAATGGAGGTT & GCATTTCCCAGATCTGTCCT & NM_009742.3 \\
\hline Clqa & CAAGGACTGAAGGGCGTGAA & CAAGCGTCATTGGGTTCTGC & NM_007572.2 \\
\hline C3 & CACCGCCAAGAATCGCTAC & GATCAGGTGTTTCAGCCGC & NM_009778.3 \\
\hline $\mathrm{Ccl} 2$ & TGCTACTCATTCACCAGCAA & GTCTGGACCCATTCCTTCTT & NM_011333.3 \\
\hline $\mathrm{Ccl} 3$ & GACAAGCTCACCCTCTGTCA & GCCGGTTTCTCTTAGTCAGG & NM_011337.2 \\
\hline $\mathrm{CCl} 4$ & AGCAACACCATGAAGCTCTG & CTGTCTGCCTCTTTTGGTCA & NM_013652.2 \\
\hline Ccla & AGTGGTCTGTGGGACTTTGG & CAGACCTGTGGCTGCATAGA & NM_011338.2 \\
\hline Cfh & GAGCCTGAGACCCAACTTCC & TGTGCAACGAAGGTAGTCCC & NM_009888.3 \\
\hline Csf1 & AGTCTGTCTTCCACCTGCTG & TTCCACCTGTCTGTCCTCAT & NM_007778.4 \\
\hline Cxcl1 & ACCCGCTCGCTTCTCTGT & AAGGGAGCTTCAGGGTCAAG & NM_008176.3 \\
\hline $\mathrm{CxCl} 2$ & CAGACTCCAGCCACACTTCA & TTCAGGGTCAAGGCAAACTT & NM_009140.2 \\
\hline $\mathrm{CxCl} 3$ & ATCCAGAGCTTGACGGTGAC & GGACTTGCCGCTCTTCAGTA & NM_203320.3 \\
\hline Cxcl10 & GCCGTCATTTTCTGCCTCATCCT & CTCATTCTCACTGGCCCGTCATC & NM_021274.2 \\
\hline Cxcl12 & CGCCAGAGCCAACGTCAAGC & TTCGGGTCAATGCACACTTG & NM_021704.3 \\
\hline Cxcr4 & GAAGTGGGGTCTGGAGACTAT & TTGCCGACTATGCCAGTCAAG & NM_009911.3 \\
\hline Ddit4 & CAAGGCAAGAGCTGCCATAG & CCGGTACTTAGCGTCAGGG & NM_029083.2 \\
\hline Fasl & CATGGAGTGGTCCTTAATGC & AAGTAGACCCACCCTGGAAG & NM_010177.4 \\
\hline Fgf2 & CACCAGGCCACTTCAAGGA & GATGGATGCGCAGGAAGAA & NM_008006.2 \\
\hline Gapdh & CCAGCTCGTCCTGTAGACAA & GCCTTGACTGTGCCGTTGA & NM_008084.3 \\
\hline Hprt & GCTGACCTGCTGGATTACAT & TTGGGGCTGTACTGCTTAAC & NM_013556.2 \\
\hline Ifng & TTCTTCAGCAACAGCAAGGC & TCAGCAGCGACTCCTTTTCC & NM_008337.4 \\
\hline$\| 1 \mathrm{a}$ & CGGGTGACAGTATCAGCAAC & GACAAACTTCTGCCTGACGA & NM_010554.4 \\
\hline$\| 1 b$ & CCCAACTGGTACATCAGCAC & TCTGCTCATTCACGAAAAGG & NM_008361.4 \\
\hline$\| 1 \mathrm{rn}$ & CCTCGGGATGGAAATCTGCTG & AGGCCTCGGCAGTACTATTGG & NM_031167.5 \\
\hline $1112 a$ & GATGACATGGTGAAGACGGC & AGGCACAGGGTCATCATCAA & NM_008351.3 \\
\hline Ltb4r1 & TGGCTGCAAACACTACATCTCCT & CACTGGCATACATGCTTATTCCAC & NM_008519.2 \\
\hline Mdk & GTCAATCACGCCTGTCCTCT & CAAGTATCAGGGTGGGGAGA & NM_010784.5 \\
\hline Mif & TGCCCAGAACCGCAACTACAGTAA & TCGCTACCGGTGGATAAACACAGA & NM_010798.3 \\
\hline Nos2 & GGCCAGCCTGTGAGACCTTT & TTGGAAGTGAAGCGTTTCG & NM_010927.4 \\
\hline Osm & CTGTAAATGGGAACCTGTGG & TTCAAGAGGACTGGTTCAGC & NM_001013365.2 \\
\hline$P 2 r \times 7$ & GGGTGGGGTGACGAAGTTAG & GGGTCCATCCACCCCTTTTTT & NM_011027.4 \\
\hline Pcna & TAAAGAAGAGGAGGCGGTAA & TAAGTGTCCCATGTCAGCAA & NM_011045.2 \\
\hline Ptgs2 & CAGACAACATAAACTGCGCCTT & GATACACCTCTCCACCAATGACC & NM_011198.4 \\
\hline Slpi & GCTGTGAGGGTATATGTGGGAAA & CGCCAATGTCAGGGATCAG & NM_011414.3 \\
\hline Tgfb1 & GCTACCATGCCAACTTCTGT & CGTAGTAGACGATGGGCAGT & NM_011577.2 \\
\hline Tnfa & СCCACTCTGACCCCTTTACT & TTTGAGTCCTTGATGGTGGT & NM_013693.3 \\
\hline Tnfaip3 & CGAGAGAGAACCCCAGAAGA & ATGCATGAGGCAGTTTCCA & NM_009397.3 \\
\hline Tnfsf10 & CCTCTCGGAAAGGGCATTC & TCCTGCTCGATGACCAGCT & NM_009425.2 \\
\hline Vegfa & AGACACACCCACCCACATAC & CAGACCACACTGAAGCCTTT & NM_009505.4 \\
\hline Vegfb & CCTCTGAGCATGGAACTCAT & TTTGGTCTGCATTCACATTG & NM_011697.3 \\
\hline
\end{tabular}

pooled blood and spinal cord neutrophil samples. Briefly, all primers were pooled and pre-amplification of each cDNA sample was performed for 10 cycles using a thermocycler. Primers and pre-amplified samples were then loaded into the gene expression array and analyzed on a Biomark HD system (Fluidigm). Delta $(\Delta) \mathrm{CT}$ values for each gene were determined by subtracting the average $C T$ value for three housekeeping genes (Gapdh, Actb, and 
Hprt1). Genes in which CT values could not be obtained for all or more than half of the biological replicates were considered not detected (n.d.).

\section{Statistical analysis}

All statistical analyses were performed using Prism Software (GraphPad). BMS scores and $\mathrm{Ly} 6 \mathrm{G}^{+}$neutrophil counts in spinal cord cross-sections were evaluated using a two-way, repeated measures ANOVA followed by Bonferroni's multiple comparisons test. Comparison between two groups for flow cytometry, white matter sparing, and immunoblots was performed using an unpaired two-tailed Student's $t$ test. A one-way ANOVA followed by Tukey's post hoc test was used for flow cytometry data comparing neutrophils from the blood and spinal cord of Syk ${ }^{\mathrm{f} / \mathrm{f}}$ and Syk ${ }^{\mathrm{f} / \mathrm{f}} \mathrm{MRP} 8$-Cre mice. To compare the proportion of animals with weight-supported stepping in the open field analysis (BMS), a chi-squared analysis was used followed by a one-sided Fisher's exact test. Cytokine expression was evaluated using a two-way ANOVA followed by two-stage step-up method of Benjamini, Krieger, and Yekutieli correction for multiple comparisons with a false discovery rate (FDR) of 0.05 . Only genes in which CT values could be determined for all three biological replicates were considered for statistical analysis (28/40 genes). Sex as a biological variable (SABV) was evaluated using a three-way, repeated measures ANOVA for BMS and a two-way ANOVA for all other assays. Significance was designated as $p$ - and $q$-values $<0.05$.

\section{Results}

Syk contributes to long-term neurologic deficits

To determine if Syk signaling in neutrophils contributes to long-term neurologic deficits after SCI, we examined functional recovery using the Basso Mouse Scale (BMS) following moderate thoracic contusion $\mathrm{SCI}$ at $\mathrm{T} 9$ in $\mathrm{Syk}^{\mathrm{f} / \mathrm{f}}$ and $\mathrm{Syk}^{\mathrm{f} / \mathrm{f}} \mathrm{MRP} 8$-Cre mice. Improved long-term recovery was observed in $\mathrm{Syk}^{\mathrm{f} / \mathrm{f}} \mathrm{MRP} 8-\mathrm{Cre}$ compared to Syk $\mathrm{f}^{\mathrm{f} / \mathrm{f}}$ littermates starting at 21 days post-SCI and continuing through the end of the study (Fig. $1 \mathrm{~A} ; p=0.01$ ). No sex-specific effects were observed $(p=0.92)$. In a secondary analysis of the BMS data, approximately $60 \%$ of

\section{A}

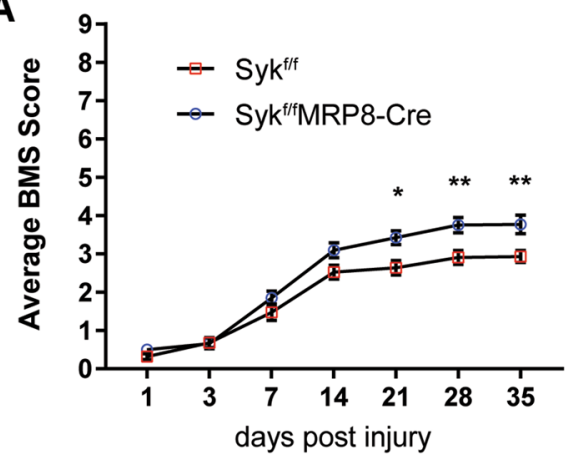

C

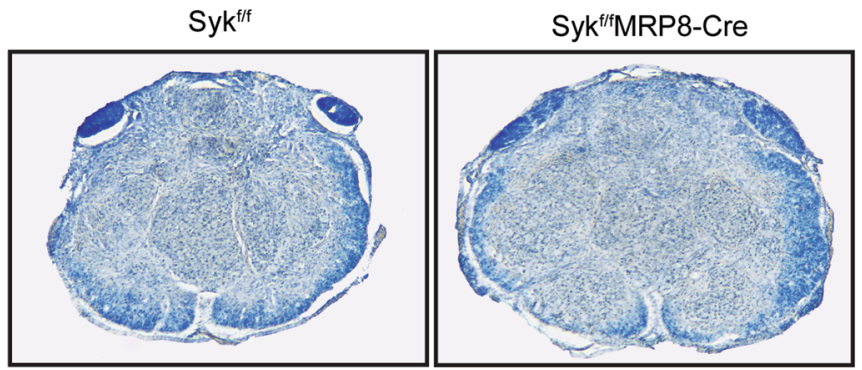

B

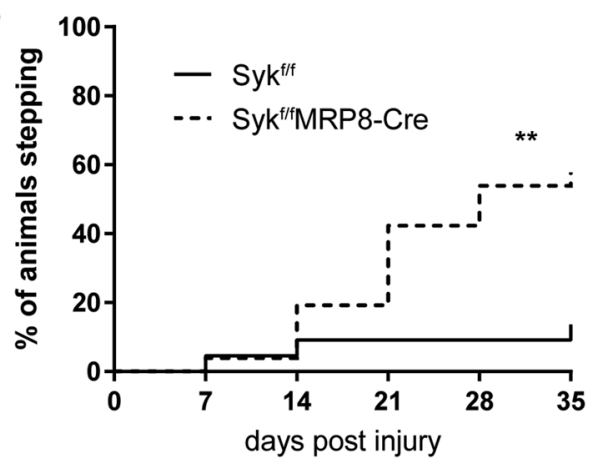

$\square$ Syk $^{\text {fff }} \circ$ Syk ${ }^{f f / 4 R P 8-C r e}$

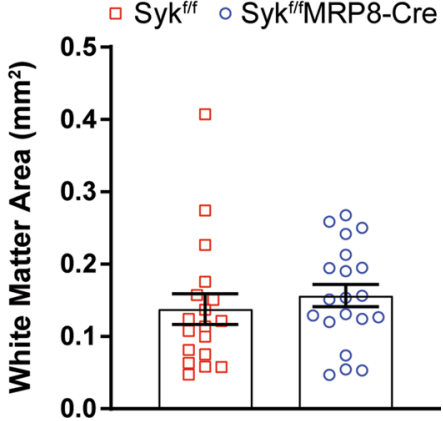

Fig. 1 Neutrophil-specific deletion of Syk enhances long-term functional recovery, but not white matter sparing by 35 days post-SCI. A Greater BMS scores were observed in Syk ${ }^{\mathrm{f} / f}$ MRP8-Cre relative to Syk ${ }^{\mathrm{f} / \mathrm{f}}$ controls starting at 21 days post-SCI. N=22 for Syk ${ }^{\mathrm{f} / \mathrm{f}}(13 \mathrm{~F} / 9 \mathrm{M}$ ) and 26 for Syk $\mathrm{flf}$ MRP8-Cre (16F/10M). Repeated measures two-way ANOVA with Bonferonni's post hoc test. B Weight-supported stepping was improved in Syk $\mathrm{F}^{\mathrm{f} / \mathrm{M}} \mathrm{MRP} 8-\mathrm{Cre}$ compared to Syk ${ }^{\mathrm{f} / \mathrm{f}}$ mice. $N=22$ for Syk $\mathrm{f}^{\mathrm{fff}}(13 \mathrm{~F} / 9 \mathrm{M})$ and 26 for Syk $/ \mathrm{f} / \mathrm{MRP} 8-C r e(16 \mathrm{~F} / 10 \mathrm{M}) \cdot X^{2}$ analysis with Fischer's exact test. C Representative images of the lesion epicenter, stained with eriochrome cyanine, in Syk ${ }^{f / f}$ and Syk ${ }^{f / f} \mathrm{MRP} 8-C r e$. No difference in white matter area was observed at 35 days post-SCI. $N=18$ for Syk ${ }^{\mathrm{f} / \mathrm{f}}(13 \mathrm{~F} / 5 \mathrm{M})$ and 20 for Syk ${ }^{\mathrm{f} / \mathrm{f}} \mathrm{MRP} 8-C r e(14 \mathrm{~F} / 6 \mathrm{M})$. Unpaired two-tailed Student's $t$ test. ${ }^{*} p<0.05,{ }^{* *} p<0.01$. SCl spinal cord injury, BMS Basso mouse scale 
Syk $^{\mathrm{f} / \mathrm{f}}$ MRP8-Cre mice obtained weight-supported stepping (BMS $\geq 4$ ), compared to $17 \%$ of Syk $^{\mathrm{f} / \mathrm{f}}$ mice, by 35 days post-SCI (Fig. 1B; $p<0.01$ ). Long-term white matter sparing after SCI was assessed between Syk $^{\mathrm{f} / \mathrm{f}}$ and $\mathrm{Syk}^{\mathrm{f} /}$ ${ }^{\mathrm{f}}$ MRP8-Cre mice in spinal cord cross-sections stained with eriochrome cyanine. No differences were observed between the two genotypes in residual white matter area at the lesion epicenter at 35 days post-SCI (Fig. 1C; $p=0.24$ ), indicating that improved functional recovery in Syk ${ }^{\mathrm{f} / \mathrm{f}} \mathrm{MRP} 8$-Cre mice was not due to greater sparing of white matter tissue at the SCI lesion site.

\section{Syk promotes neutrophil activation in the injured spinal cord}

We performed flow cytometry analysis of injured spinal cords from Syk ${ }^{\mathrm{f} / \mathrm{f}}$ and Syk ${ }^{\mathrm{f} / \mathrm{f}} \mathrm{MRP} 8$-Cre mice at 1 days postSCI to assess if Syk mediates neutrophil accumulation. Total leukocyte and myeloid cell populations were first identified with CD45 and CD11b antibodies, respectively. Myeloid cells were further separated into Ly6G ${ }^{+}$(neutrophils) and $\mathrm{Ly} 6 \mathrm{G}^{-}$(monocyte) populations (Fig. 2A). In the spinal cord samples, monocyte and microglia populations were subdivided based on CD $45^{\text {hi }}$ vs CD $45^{\text {low }}$ levels, respectively. Intracellular staining for Syk was performed to determine the efficiency of the conditional knockout in circulating and infiltrated $\mathrm{Ly}_{6 \mathrm{G}}{ }^{+}$neutrophils at 1 days post-SCI. In Syk ${ }^{\mathrm{f} / \mathrm{f}}$ mice, most Ly6G ${ }^{+}$neutrophils $(>90 \%)$ showed positive immunolabeling for Syk, whereas few $\mathrm{Ly} 6 \mathrm{G}^{+} / \mathrm{Syk}^{+}$neutrophils $(\sim 5 \%)$ were detected in $\mathrm{Syk}^{\mathrm{f} /}$ ${ }^{\mathrm{f}}$ MRP8-Cre mice (Fig. 2B; $p<0.001$ ). There was a modest decrease in $\mathrm{Ly} 6 \mathrm{G}^{+} / \mathrm{Syk}^{+}$neutrophils in the spinal cord compared to the blood of Syk ${ }^{\mathrm{f} / \mathrm{f}}$ mice $(96.3 \%$ vs. $91.1 \%$, $p=0.02$ ). No differences were observed between $\mathrm{Syk}^{\mathrm{f} / \mathrm{f}}$ and $\mathrm{Syk}^{\mathrm{f} / \mathrm{f}} \mathrm{MRP} 8-\mathrm{Cre}$ mice in the proportion of circulating $\mathrm{CD}_{11} \mathrm{~b}^{+}$myeloid cells or myeloid subsets relative to the total $\mathrm{CD} 45^{+}$leukocyte population (Fig. 2C; $p>0.22$ for all cell types). Furthermore, there were no differences in the number of accumulated $\mathrm{CD} 45^{+}$leukocytes or leukocyte subsets, including $\mathrm{Ly}_{6 \mathrm{G}}{ }^{+}$neutrophils, in the acutely injured spinal cord (Fig. 2D; $p>0.39$ for all cell types). We also performed semi-automated counting of Ly6G ${ }^{+}$ neutrophils in spinal cord cross-sections at every $500 \mu \mathrm{m}$ across the SCI lesion ( $3 \mathrm{~mm}$ total) at 1 days post-SCI. No differences in the number of $\mathrm{Ly}_{6 \mathrm{G}}{ }^{+}$neutrophils were observed across the SCI lesion (Fig. 2E; $p=0.19$ ) or in the spinal cord grey matter (Fig. 2F; $p=0.34$ ), white matter (Fig. 2G; $p=0.89$ ), or spinal meninges (Fig. $2 \mathrm{H} ; p=0.33$ ).

To determine if the genetic loss of Syk affects neutrophil activation, we also performed flow cytometry analysis of L-selectin levels (CD62L G-MFI) on circulating and infiltrated leukocyte populations at 1 days post-SCI. Following activation, L-selectin undergoes proteolytic cleavage resulting in "shedding" of the ligand-binding ectodomain $[49,50]$. Reduced L-selectin levels, therefore, are commonly used as an indicator of activation in leukocyte populations. We observed high, yet similar, levels of $\mathrm{L}$-selectin on circulating $\mathrm{CD} 11 \mathrm{~b}^{+}$myeloid cells, $\mathrm{Ly}_{6 \mathrm{G}}{ }^{+}$neutrophils, and $\mathrm{Ly}_{6 \mathrm{G}}{ }^{-}$monocyte populations from injured $\mathrm{Syk}^{\mathrm{f} / \mathrm{f}}$ and $\mathrm{Syk}^{\mathrm{f} / \mathrm{f}} \mathrm{MRP} 8-\mathrm{Cre}$ mice (Fig. 3A; $p>0.05$ for all cell types). While lower L-selectin levels were observed on infiltrated leukocytes populations, $\mathrm{Ly}_{6 \mathrm{G}}{ }^{+}$neutrophils from the injured spinal cords of $\mathrm{Syk}^{\mathrm{f} / \mathrm{f}} \mathrm{MRP} 8$-Cre mice had $\sim 36 \%$ greater L-selectin levels relative to $\mathrm{Syk}^{\mathrm{f} / \mathrm{f}}$ mice indicative of reduced activation (Fig. 3B; $p=0.04$ ). No sex-specific effects were observed $(p=0.84)$. No differences in L-selectin levels were observed for $\mathrm{CD} 45^{\text {hi }} / \mathrm{Ly}_{6} \mathrm{G}^{-}$monocytes or CD $45^{\text {low }} /$ Ly6G ${ }^{-}$microglia in the acutely injured spinal cords of $\mathrm{Syk}^{\mathrm{f} / \mathrm{f}}$ and Syk ${ }^{\mathrm{f} / \mathrm{f}} \mathrm{MRP} 8-C r e$ mice $(p=0.93$ and 0.72 , respectively).

\section{Syk does not facilitate common neutrophil effector functions acutely after $\mathrm{SCl}$}

Activated neutrophils can employ a wide range of responses depending on the encountered stimuli, although the specific effector functions utilized in the injured spinal cord have yet to be determined. To assess the effect of Syk-deletion on neutrophil effector functions acutely after SCI, we performed flow cytometry analysis of degranulation, ROS production, and NET formation on accumulated neutrophils acutely after SCI. There was a marked increase in all three effector functions in infiltrated neutrophils relative to their circulating counterparts at 1 days post-SCI (Fig. 3C-E; $p<0.01$ ). No differences in the levels of ROS labeling (DHR123 G-MFI) were observed for $\mathrm{Ly}_{6 \mathrm{G}}{ }^{+}$neutrophils in the blood or

(See figure on next page.)

Fig. 2 Syk-deficiency does not alter neutrophil accumulation in the acutely injured spinal cord. A Flow cytometry gating of leukocytes (CD45 $5^{+}$, myeloid lineage cells $\left(\mathrm{CD} 11 \mathrm{~b}^{+}\right)$, neutrophils $\left(\mathrm{Ly}_{6} \mathrm{C}^{\text {low }} / \mathrm{Ly} 6 \mathrm{G}^{+}\right)$, and monocytes $\left(\mathrm{Ly} 6 \mathrm{G}^{-}\right)$in the blood and spinal cord at 1 days post-SCl. Microglia were identified in the spinal cord as CD1 1 b ${ }^{+} / \mathrm{Ly}_{6 \mathrm{G}^{-}} / \mathrm{CD} 45^{\text {low }}$ cells. B Neutrophils from the blood and spinal cord of Syk ${ }^{\mathrm{f} / \mathrm{M}}$ MRP8-Cre show robust Syk-deficiency relative to Syk ${ }^{f / f}$ controls at 1 days post-SCI. Two-way ANOVA with Tukey's post hoc test. C Syk-deficiency does not alter the proportion of myeloid lineage cells in the peripheral blood compartment at 1 days post-SCI. D No differences were observed in the infiltration of leukocytes or myeloid lineage subtypes at 1 days post-SCI. $N=7$ for Syk ${ }^{f / f}(4 F / 3 M)$ and 6 for Syk $k^{f / f}$ MRP8-Cre (3F/3M) for B-D. Unpaired two-tailed Student's $t$ test for C-D. E No differences were observed in the number of neutrophils (Ly6G $\mathrm{G}^{+}$cells) in sections spanning the lesion site in Syk/f and Syk ${ }^{\mathrm{f} / \mathrm{A}} \mathrm{MRP} 8-\mathrm{Cre}$ mice. F-H No differences were found in the accumulation of neutrophils in the grey matter, white matter, or meninges of Syk $\mathrm{k}^{\mathrm{f} / \mathrm{f}}$ and Syk ${ }^{\mathrm{f} / \mathrm{f}} \mathrm{MRP} 8$-Cre mice. $N=5$ for Syk ${ }^{\mathrm{f} / \mathrm{f}}(3 \mathrm{~F} / 2 \mathrm{M})$ and 5 for Syk $\mathrm{k}^{\mathrm{f} / \mathrm{f}} \mathrm{MRP} 8-\mathrm{Cre}(2 \mathrm{~F} / 3 \mathrm{M})$ for E-H. Repeated measures two-way ANOVA with Bonferonni's post hoc test. ${ }^{*} p<0.05,{ }^{* * *} p<0.001$. SCl spinal cord injury 
A
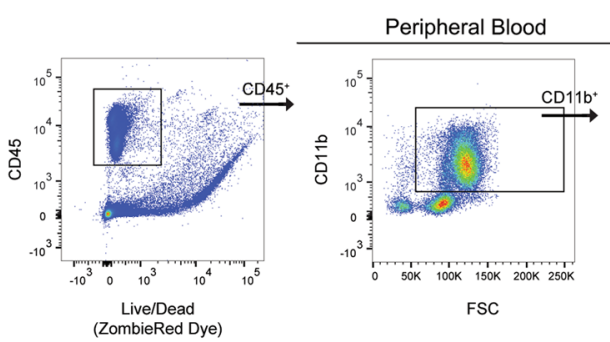

FSC

Spinal Cord
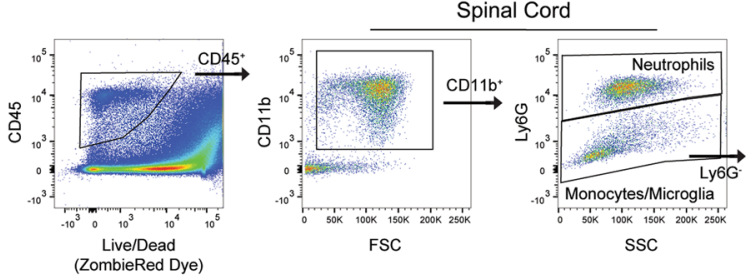
(ZombieRed Dye)

C

Peripheral Blood

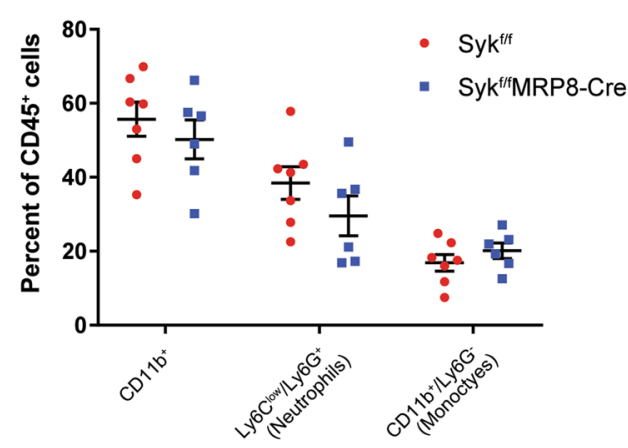

E

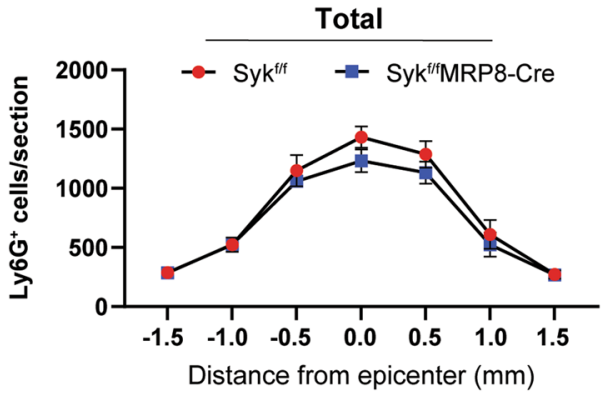

G

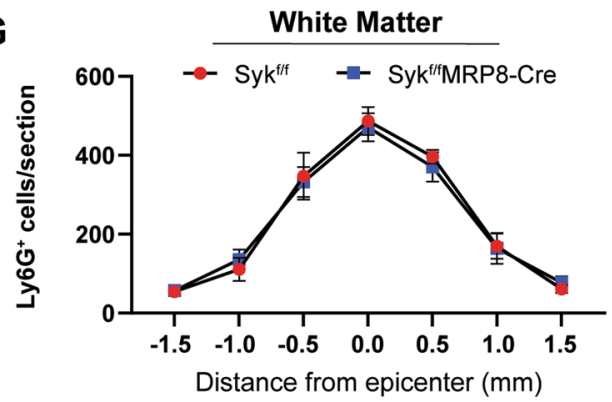

F

H
B
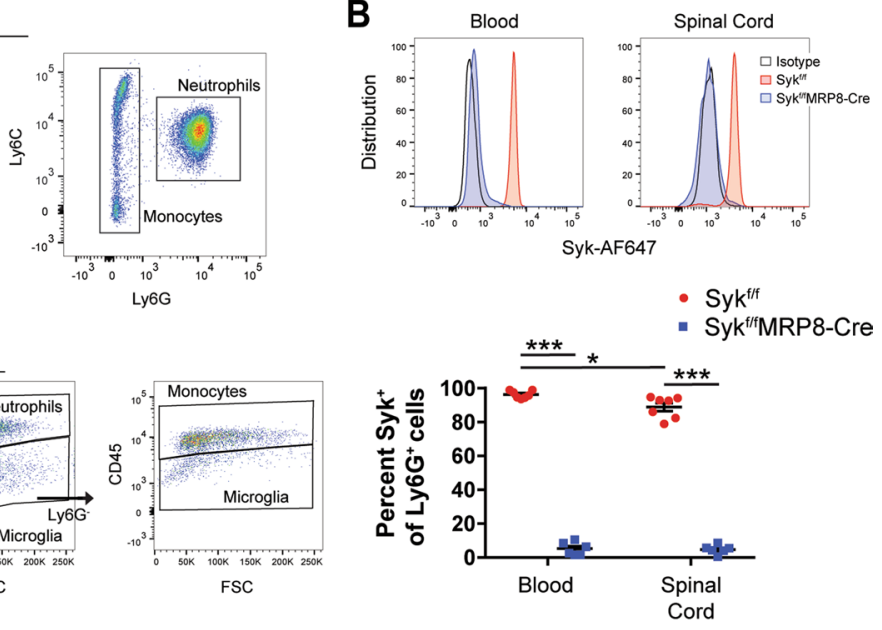

D
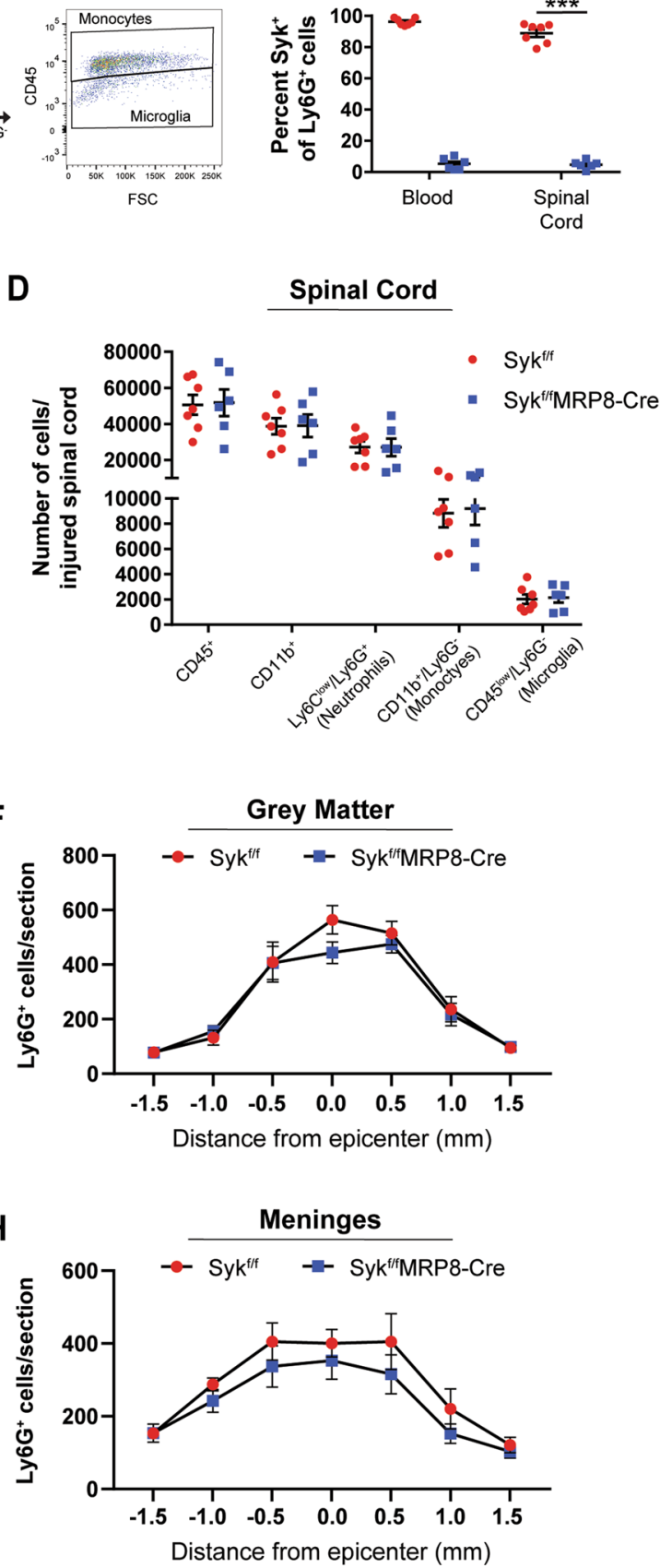

Fig. 2 (See legend on previous page.) 


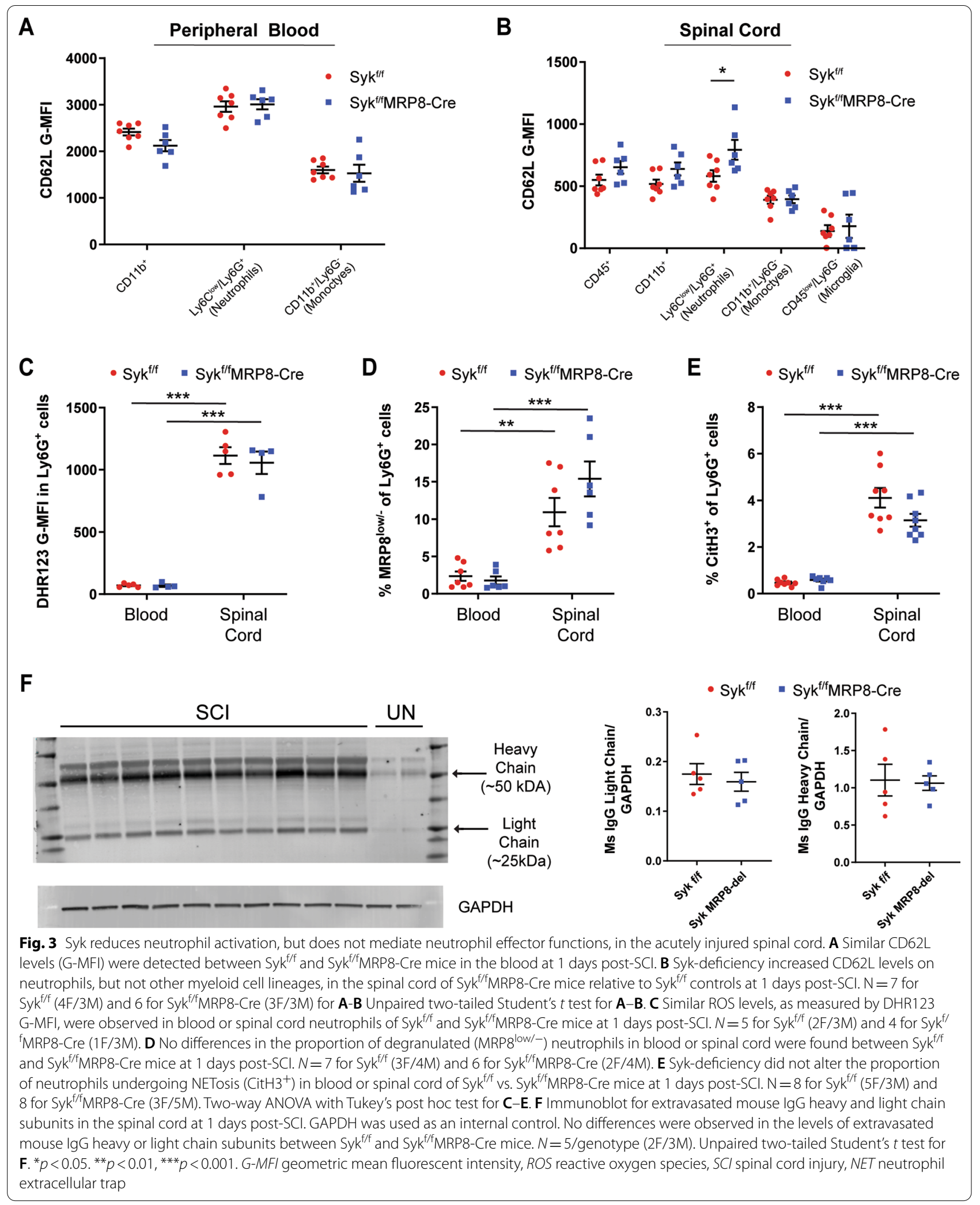



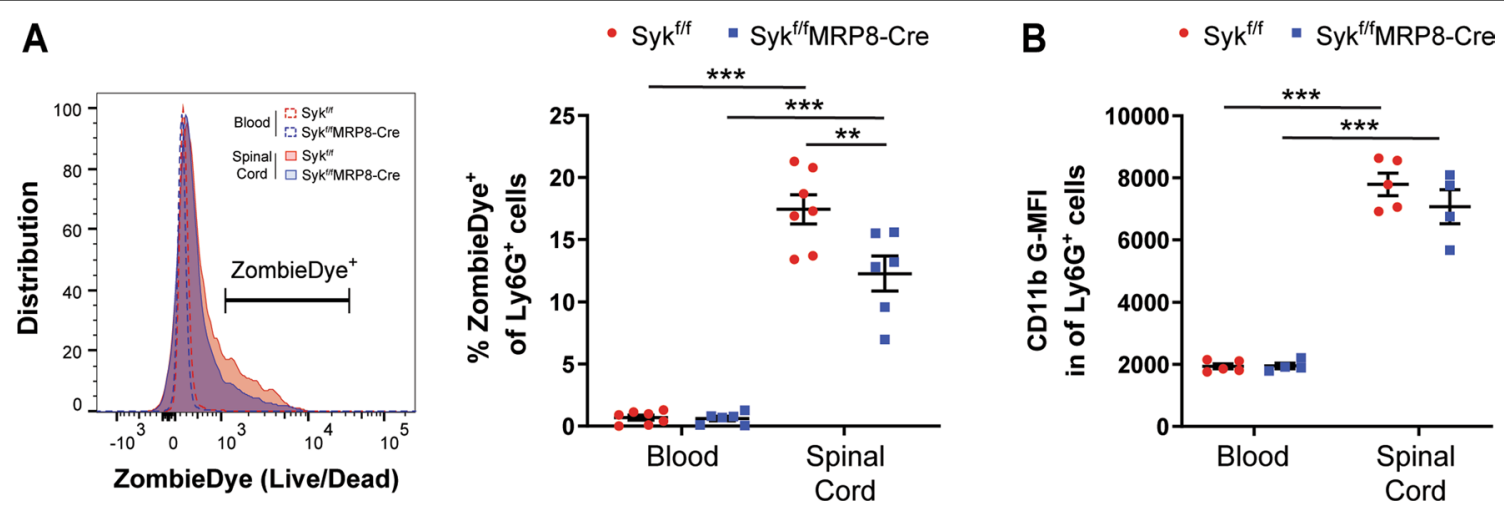

Fig. 4 Syk augments neutrophil cell death in the acutely injured spinal cord independent of CD 11 b levels. A Representative flow cytometry histograms of ZombieDye levels in blood and spinal cord neutrophils. Syk deficiency reduced the proportion of intraspinal neutrophils labeled

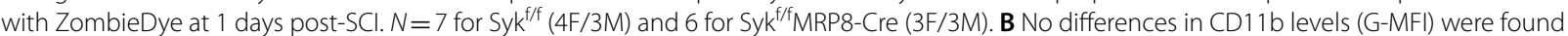
between Syk ${ }^{\mathrm{f} / \mathrm{f}}$ and Syk ${ }^{\mathrm{f} / \mathrm{M}} \mathrm{MRP} 8-\mathrm{Cre}$ mice at 1 days post-SCI. $N=5$ for Syk ${ }^{\mathrm{f} / \mathrm{f}}(2 \mathrm{~F} / 3 \mathrm{M})$ and 4 for Syk ${ }^{\mathrm{f} / \mathrm{M}} \mathrm{MRP} 8-C r e(1 \mathrm{~F} / 3 \mathrm{M})$. Two-way ANOVA with Tukey's post hoc test for $\mathbf{A}, \mathbf{B} .{ }^{* *} p<0.01,{ }^{* *} p<0.001$. G-MFI geometric mean fluorescent intensity, SCl spinal cord injury

spinal cord of Syk ${ }^{\mathrm{f} / \mathrm{f}}$ vs. Syk ${ }^{\mathrm{f} / \mathrm{f}}$ MRP8-Cre mice (Fig. 3C; $p=0.99$ and 0.88 , respectively). There were no differences observed in the proportion of $\mathrm{Ly}_{6 \mathrm{G}} \mathrm{G}^{+}$neutrophils with diminished MRP8 levels (Ly6G $\left.{ }^{+} / \mathrm{MRP}^{\text {low/-}}\right)$, indicative of degranulation, in the blood or injured spinal cords of $\mathrm{Syk}^{\mathrm{f} / \mathrm{f}}$ relative to $\mathrm{Syk}^{\mathrm{f} / \mathrm{f}} \mathrm{MRP} 8-C r e$ mice (Fig. 3D; $p=0.99$ and 0.21 , respectively). We also observed no differences in the proportion of $\mathrm{Ly}_{6 \mathrm{G}}^{+}$neutrophils undergoing NETosis $\left(\mathrm{Ly}_{6 \mathrm{G}}{ }^{+} / \mathrm{CitH} 3^{+}\right)$in the blood or spinal cords of $\mathrm{Syk}^{\mathrm{f} / \mathrm{f}} \mathrm{MRP} 8-\mathrm{Cre}$ compared to $\mathrm{Syk}^{\mathrm{f} / \mathrm{f}}$ mice at 1 days postSCI (Fig. 3E; $p=0.99$ and 0.06 , respectively).

Since there was minimal effect of Syk deficiency on the cytotoxic effector functions utilized by neutrophils in the acutely injured spinal cord, we sought to determine if there were any differences in acute secondary injury by assessing the accumulation of endogenous mouse IgG antibody, which can extravasate from the blood plasma into the spinal cord during blood-spinal cord barrier disruption [51]. We found no differences in accumulated mouse IgG light and heavy chain subunits, measured by immunoblotting, in the spinal cords of Syk ${ }^{\mathrm{f} / \mathrm{f}} \mathrm{MRP} 8-\mathrm{Cre}$ mice relative to $\mathrm{Syk}^{\mathrm{f} / \mathrm{f}}$ mice at 1 days post-SCI (Fig. 3F; $p=0.60$ and 0.87 , respectively) indicating that Syk in neutrophils does not contribute to acute blood-spinal cord barrier disruption.

\section{Syk augments neutrophil cell death in the acutely injured spinal cord}

While apoptosis has often been described as an antiinflammatory cell fate pathway for activated neutrophils, studies have shown that dead neutrophils are not efficiently cleared in SCI [26, 27]. To determine if Syk contributes to neutrophil cell death in the injured spinal cord, we incubated dissociated spinal cord samples with a cell-impermeant, amine-fixable dye (ZombieDye) that brightly labels dead cells with compromised membrane integrity. While there was a substantial increase in ${\text { Ly } 6 G^{+}}^{+}$ZombieDye ${ }^{+}$neutrophils in the injured spinal cord relative to the blood at 1 days post-SCI (Fig. 4A; $p<0.001$ ), we observed a reduced proportion of $\mathrm{Ly} 6 \mathrm{G}^{+} /$ ZombieDye $^{+}$neutrophils in the injured spinal cords of $\mathrm{Syk}^{\mathrm{f} / \mathrm{f}} \mathrm{MRP} 8-C r e$ relative to $\mathrm{Syk}^{\mathrm{f} / \mathrm{f}}$ mice at 1 days post-SCI (12.3\% vs. $17.4 \%$, respectively; $p=0.003)$. No sex-specific effects were observed $(p=0.88) . \beta_{2}$ integrins, such as Mac-1 (CD11b/CD18), have been shown to contribute to neutrophil apoptosis [52], therefore, we also assessed the surface levels of CD11b on neutrophils by flow cytometry. While there was a marked increase in CD11b levels on $\mathrm{Ly} 6 \mathrm{G}^{+}$neutrophils in the spinal cord relative to the blood $(p<0.001)$, we found no differences in CD11b G-MFI on Ly6G ${ }^{+}$neutrophils in the blood or spinal cord between $\mathrm{Syk}^{\mathrm{f} / \mathrm{f}}$ and Syk ${ }^{\mathrm{f} / \mathrm{f}}$ MRP8-Cre mice (Fig. 4B; $p=0.99$ and 0.41 , respectively).

\section{Syk contributes to cytokine expression in neutrophils after $\mathrm{SCl}$}

We have previously shown that Syk signaling contributes to cytokine secretion in stimulated neutrophils in vitro [38], however, the role of Syk in the upregulation of cytokines and chemokines by neutrophils after SCI remains unknown. Furthermore, changes in the expression of cytokines and chemokines by neutrophils following infiltration into the acutely injured spinal cord have yet to be determined. To assess whether SCI and Syk signaling contribute to the expression of cytokines, chemokines, and other inflammatory mediators in neutrophils, we performed qPCR analysis of 40 genes (Table 1) using a gene array on mRNA from neutrophils 
isolated from the spinal cord and blood at 1 days post$\mathrm{SCI}$, as well as from the blood of uninjured Syk ${ }^{\mathrm{f} / \mathrm{f}}$ mice. Magnetic isolation was sufficient to achieve $>99 \%$ purity of $\mathrm{Ly} 6 \mathrm{G}^{+}$neutrophils from the blood and spinal cord (Fig. 5A) for mRNA isolation and analysis. We observed widespread changes in transcripts levels $(\triangle \mathrm{CT})$ for many of the genes in our panel (Fig. 5B). Transcripts for several genes (Arg1, C1qa, Fgf2, and Cxcl1) could not be detected in blood neutrophils, but were highly upregulated in neutrophils in the spinal cords of both $\operatorname{Syk}^{\mathrm{f} / \mathrm{f}}$ and Syk ${ }^{\mathrm{f} / \mathrm{f}} \mathrm{MRP} 8$ Cre mice. Ifng mRNA was not detected in either blood or spinal cord neutrophils.

Of the 40 genes in our panel, we performed statistical analysis on the 28 genes in which CT values could be detected for all samples (Table 2). Among these genes, we observed upregulation of $\mathrm{Ccl} 3$ and $\mathrm{Cxcl} 2$ in the blood of $\mathrm{Syk}^{\mathrm{f} / \mathrm{f}}$ mice acutely following SCI relative to uninjured controls. We found marked upregulation of several cytokines, chemokines, and inflammation-related genes (Bcl2a1, Ccl3, Ccl4, Ccl9, Csf1, Cxcl2, Cxcl3, Cxcl10, Ddit4, Il1a, Il1rn, Mdk, Mif, P2rx7, Ptgs2, Tnfa, Tnfaip3, and $V e g f a$ ) in infiltrated neutrophils relative to circulating neutrophils in both $\mathrm{Syk}^{\mathrm{f} / \mathrm{f}}$ and Syk ${ }^{\mathrm{f} / \mathrm{f}} \mathrm{MRP}$ 8-Cre mice. Of the genes evaluated in our array, only Slpi was downregulated in infiltrated neutrophils relative to circulating neutrophils in both genotypes. When comparing between Syk $^{\mathrm{f} / \mathrm{f}} \mathrm{MRP} 8-C r e$ and Syk ${ }^{\mathrm{f} / \mathrm{f}}$ mice, we observed downregulation of Csf1 (3.9-fold) and Vegfa (3.4-fold) in infiltrated neutrophils from $\mathrm{Syk}^{\mathrm{f} / \mathrm{f}} \mathrm{MRP} 8-\mathrm{Cre}$ at 1 days post-SCI $(q=0.009$ and 0.02 , respectively). Il1 $a$ was upregulated 3.1 -fold in the blood of $\mathrm{Syk}^{\mathrm{f} / \mathrm{f}} \mathrm{MRP} 8$-Cre mice relative to $\mathrm{Syk}^{\mathrm{f} / \mathrm{f}}$ controls at 1 days post-SCI $(q=0.03)$. Our findings indicate that Syk signaling contributes to the expression of specific cytokines in neutrophils after SCI.

\section{Discussion}

Tyrosine kinases, including Syk, help coordinate the complex intracellular signaling pathways downstream of immunoreceptors in neutrophils to enable appropriate cellular responses to the extracellular cues sensed at injury and infection sites [32, 33]. However, relatively little is known about the functional responses of neutrophils in the injured spinal cord or the role of Syk in mediating pathogenic neutrophil activities after SCI. Here we show that Syk augments neutrophil activation, cell death, and chemokine expression, but does not mediate the development of neutrophil effector functions within the acutely injured spinal cord. We demonstrate the neutrophil-specific deletion of Syk improves long-term functional recovery, but does not alter long-term white matter sparing. Our findings indicate that Syk mediates pathogenic neutrophil activities in the injured spinal cord that exacerbate long-term neurologic deficits through

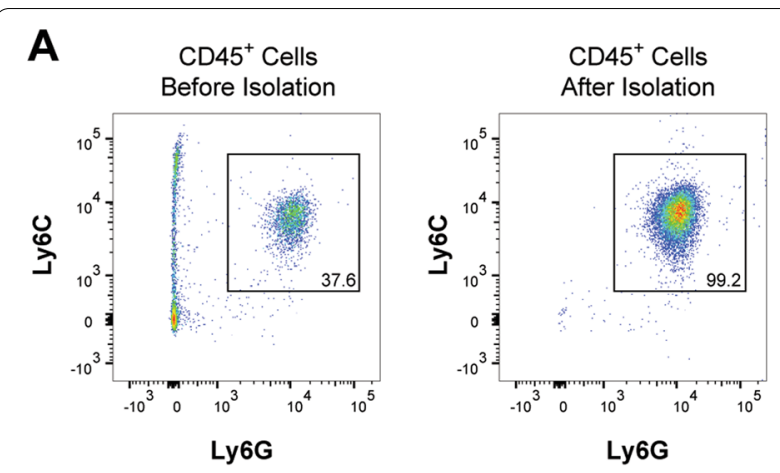

B

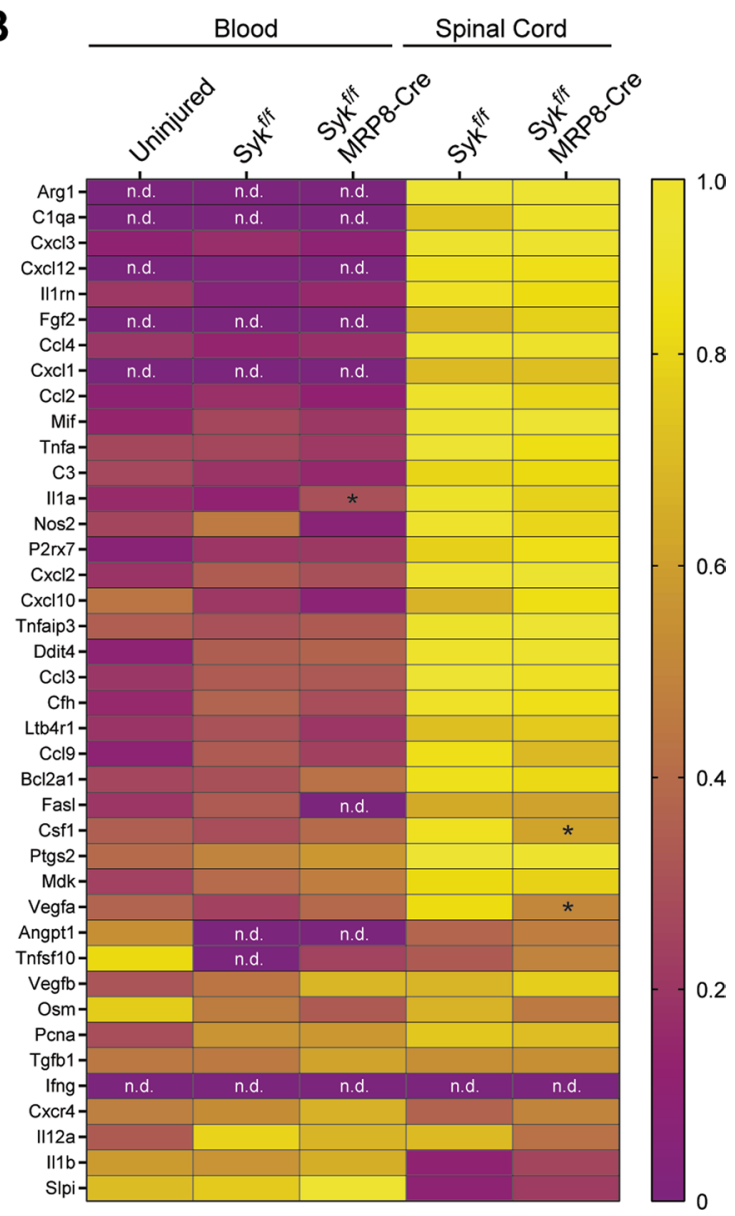

Fig. 5 Syk deficiency partially attenuates the widespread upregulation of cytokines, chemokines, and other inflammatory mediators in infiltrated neutrophils in the acutely injured spinal cord. A Magnetic isolation enhanced neutrophil purity (> 99\%) for qPCR analysis. B Heatmap of the average normalized CT value for 40 genes in neutrophils from the blood of uninjured Syk ${ }^{\mathrm{f} / \mathrm{f}}$, as well as the blood and spinal cords of Syk ${ }^{f / f}$ and Syk ${ }^{f / f}$ MRP8-Cre mice at 1 days post-SCl. Gapdh, Actb, and Hprt1 were used as internal controls. $N=3$ for uninjured $(2 F / 1 M), 3$ for Syk ${ }^{f / f}(1 F / 2 M)$, and 3 for Syk ${ }^{f /}$ ${ }^{f} \mathrm{MRP} 8-\mathrm{Cre}(\mathrm{OF} / 3 \mathrm{M})$. n.d. not detected in at least $2 / 3$ samples. Two-way ANOVA with Benjamini, Krieger, and Yekutieli multiple comparisons correction. ${ }^{*} q<0.05$ relative to Syk ${ }^{\mathrm{f} / \mathrm{f}}$. SCl spinal cord injury) 
Table 2 Gene expression $(\triangle C T)$ in blood and spinal cord neutrophils

\begin{tabular}{|c|c|c|c|c|c|c|}
\hline \multirow[t]{2}{*}{ Gene symbol } & \multicolumn{3}{|c|}{ Syk $^{\mathrm{f} / \mathrm{f}} \Delta C T$ values } & \multicolumn{2}{|c|}{$\mathrm{Syk}^{\mathrm{f} / \mathrm{f}} \mathrm{MRP} 8-\mathrm{Cre} \Delta C T$ values } & \multirow[t]{2}{*}{ FDR $q$ value } \\
\hline & UN & Blood & Spinal Cord & Blood & Spinal cord & \\
\hline $\mathrm{Bcl} 2 \mathrm{a} 1$ & 9.22 & 8.86 & 4.18 & 7.80 & 4.53 & ${ }^{\mathrm{b}} q<0.001$ \\
\hline C3 & 1.52 & 1.72 & 0.01 & 1.83 & -0.05 & \\
\hline $\mathrm{CCl} 3$ & 10.11 & 8.43 & 1.14 & 8.53 & 1.82 & ${ }^{\mathrm{b}} q<0.001,{ }^{\mathrm{d}} q=0.026$ \\
\hline $\mathrm{CCl} 4$ & 7.11 & 7.61 & 1.68 & 7.3 & 1.75 & ${ }^{\mathrm{b}} q<0.001$ \\
\hline Ccl9 & 10.55 & 9.37 & 6.84 & 9.82 & 7.58 & ${ }^{\mathrm{b}} q<0.01$ \\
\hline Cox2 & 8.98 & 8.36 & 5.67 & 7.84 & 5.49 & ${ }^{\mathrm{b}} q<0.01$ \\
\hline Csf1 & 9.93 & 10.45 & 5.78 & 9.58 & 7.76 & ${ }^{\mathrm{b}} q<0.05,{ }^{\mathrm{a}} q=0.005$ \\
\hline $\mathrm{CxCl} 2$ & 10.00 & 8.31 & 1.82 & 8.83 & 2.30 & ${ }^{\mathrm{b}} q<0.001,{ }^{\mathrm{d}} q=0.025$ \\
\hline $\mathrm{CxCl} 3$ & 11.03 & 10.18 & -0.23 & 11.12 & 0.25 & ${ }^{\mathrm{b}} q<0.001$ \\
\hline Cxcl10 & 8.38 & 9.49 & 7.27 & 10.08 & 6.47 & ${ }^{\mathrm{b}} q<0.01$ \\
\hline Cxcr4 & 0.81 & 0.63 & 1.19 & 0.16 & 0.73 & \\
\hline Ddit4 & 8.45 & 7.38 & 4.69 & 7.27 & 4.98 & ${ }^{\mathrm{b}} q<0.01$ \\
\hline ॥11a & 14.38 & 14.83 & 8.14 & 13.18 & 8.95 & ${ }^{\mathrm{b}} q<0.001,{ }^{\mathrm{c}} q=0.013$ \\
\hline$\| 1 \mathrm{~b}$ & -1.50 & -1.43 & -0.12 & -1.70 & -0.56 & \\
\hline Il1 rn & 2.71 & 3.41 & -0.10 & 2.94 & 0.07 & ${ }^{b} q<0.001$ \\
\hline$\| 12 a$ & 10.78 & 8.85 & 9.26 & 9.34 & 10.41 & \\
\hline Ltb4r1 & 2.79 & 2.50 & 1.43 & 2.77 & 1.34 & \\
\hline Mdk & 12.69 & 11.70 & 9.06 & 11.25 & 9.28 & ${ }^{\mathrm{b}} q<0.01$ \\
\hline Mif & 4.05 & 3.59 & 0.95 & 3.80 & 1.07 & ${ }^{\mathrm{b}} q<0.001$ \\
\hline Osm & 9.72 & 10.40 & 9.95 & 10.70 & 10.43 & \\
\hline$P 2 r \times 7$ & 12.61 & 12.22 & 10.43 & 12.18 & 10.23 & ${ }^{\mathrm{b}} q<0.05$ \\
\hline Pcna & 6.71 & 5.99 & 5.49 & 5.96 & 5.61 & \\
\hline Slpi & -0.77 & -0.97 & 1.19 & -1.50 & 0.78 & ${ }^{b} q<0.01$ \\
\hline Tgfb 1 & 4.26 & 4.24 & 3.99 & 3.74 & 3.97 & \\
\hline Tnfa & 8.43 & 8.44 & 5.36 & 8.68 & 5.76 & ${ }^{b} q<0.001$ \\
\hline Tnfaip3 & 6.89 & 7.08 & 4.33 & 6.93 & 4.24 & ${ }^{\mathrm{b}} \mathrm{q}<0.001$ \\
\hline Vegfa & 11.77 & 12.48 & 9.29 & 11.68 & 11.04 & ${ }^{\mathrm{b}} q<0.01,{ }^{\mathrm{a}} q=0.021$ \\
\hline Vegfb & 8.21 & 7.99 & 7.58 & 7.57 & 7.42 & \\
\hline
\end{tabular}

Values represent average $\triangle C T$ for each group. For each sample, CT values were normalized to the average of Gapdh, Actb, and Hprt1. $N=3 /$ group. Two-way ANOVA with Benjamini, Krieger, and Yekutieli multiple comparisons correction

FDR false discovery rate

${ }^{\mathrm{a}} q<0.05$ for spinal cord neutrophils between genotypes

${ }^{\mathrm{b}} q<0.05$ for blood neutrophils vs. spinal cord neutrophils (Syk ${ }^{\mathrm{f} / \mathrm{f}}$ and Syk ${ }^{\mathrm{f} / \mathrm{f}} \mathrm{MRP} 8 \mathrm{Cre}$ mice)

${ }^{c} q<0.05$ for blood neutrophils between genotypes

${ }^{\mathrm{d}} q<0.05$ for UN blood neutrophils vs. SCI blood neutrophils (Syk ${ }^{\mathrm{f} / \mathrm{f}}$ mice)

mechanisms independent of long-term white matter tissue loss.

Reducing neutrophil accumulation into the acutely injured spinal cord, by targeting adhesion receptors or inflammatory mediators involved in the recruitment of neutrophils, has predominantly led to improved tissue sparing and long-term functional recovery $[4,7-10,46]$. In our study, we demonstrate that Syk signaling does not affect neutrophil accumulation or distribution at 1 days post-SCI, which is the time point when neutrophil numbers have been previously reported to peak in murine models of SCI $[4,7,12,13]$. Our results are consistent with studies in other models of inflammation that have shown that Syk does not substantially contribute to neutrophil migration or recruitment $[38,53]$. However, we still observed improved long-term recovery in $\mathrm{Syk}^{\mathrm{f} /}$ ${ }^{\mathrm{f}}$ MRP8-Cre mice, demonstrating that Syk signaling in neutrophils mediates neurologic deficits after contusive SCI. To the best of our knowledge, this is the first report of improved long-term recovery after SCI by disrupting neutrophil function independent of recruitment. Interestingly, the improved behavioral outcomes did not coincide with long-term white matter sparing in our study. Furthermore, we did not observe any differences 
in blood-spinal cord barrier disruption in Syk-deficient mice, as measured by the extravasation of endogenous mouse antibody at 1 days post-SCI. These results indicate that Syk does not mediate neutrophil functions involved in secondary tissue damage, but could contribute to other neutrophil \& inflammatory processes that impair regeneration, neural circuit reorganization, or neuronal activity. In addition, Syk signaling may contribute to neutrophil function outside of the spinal cord and promote systemic pathological conditions, such as the development of non-alcoholic steatohepatitis [54], that could exacerbate neurologic deficits.

Recent studies have shown that neutrophils acquire distinct functional phenotypes in different tissue environments [55], however, the pathogenic activities of neutrophils within the injured spinal cord have yet to be determined. We found that Syk-deficient neutrophils acquire lower levels of activation in the acutely injured spinal cord, as indicated by greater CD62L (L-selectin) levels at 1 days post-SCI. These results are consistent with our past study that demonstrated reduced L-selectin shedding following stimulation of Syk-deficient neutrophils in vitro [38]. We also evaluated the major effector functions utilized by activated neutrophils in the injured spinal cord. While we observed substantial increases in ROS production, degranulation, and NETosis in infiltrated neutrophils relative to their circulating counterparts, we did not see any attenuation of neutrophil effector functions with Syk deficiency. These findings differ from our previous results, which demonstrated that ROS production or degranulation are augmented by Syk signaling in response to microbial stimuli in vitro [38]. One limitation in our current study is that we were only able to measure intracellular ROS production in infiltrated neutrophils ex vivo. We cannot exclude the possibility of altered extracellular release of ROS in Sykdeficient neutrophils or artifacts introduced by the tissue dissociation and the brief culture of infiltrated neutrophils ex vivo. Regardless, our results suggest that Syk does not substantially impact neutrophil effector functions in the acutely injured spinal cord. These findings, combined with the similar infiltration of Syk-deficient neutrophils into the injured spinal cord, might explain why no differences were observed in acute blood-spinal cord barrier disruption or long-term white matter sparing. Future work is needed to determine the role of Syk signaling in neutrophils that are present in the injured spinal cord after 1 days post-SCI.

While neutrophils typically express lower levels of cytokines relative to other immune cell types, their large numbers can still influence the cytokine and chemokine profiles within the injury site and profoundly shape the course of inflammation [28, 29].
We previously found that Syk can potentiate cytokine secretion by neutrophils following stimulation with $S$. aureus or E. coli in vitro [38]. Cytokine expression by neutrophils in the injured spinal cord, however, has yet to be described. In this study, we developed a 40-gene panel to broadly screen for cytokines, chemokines, and other inflammatory mediators that have been previously shown to be expressed by neutrophils in other models of inflammation or infection [28-31]. We found that activated neutrophils in the spinal cord of both genotypes markedly upregulated many of the genes assessed in our panel relative to their circulating counterparts. Interestingly, we found that neutrophils in the spinal cord downregulate SLPI, a serine protease inhibitor that has been previously shown to promote recovery after SCI [56]. To the best of our knowledge, this is the first study to broadly assess the expression of cytokine and chemokine genes in neutrophils isolated from the acutely injured spinal cord. We observed that Syk-deficient neutrophils in the injured spinal cord have lower Csf1 transcript levels relative to control Syk ${ }^{\mathrm{f} / \mathrm{f}}$ mice at 1 days post-SCI. Csf1 is a prominent chemokine involved in the proliferation and polarization of macrophages and microglia with detrimental effects in SCI and peripheral nerve injury $[57,58]$. Our findings suggest that Syk signaling can facilitate the production of chemokines that may impact subsequent inflammation or tissue repair.

Neutrophil apoptosis is typically considered an inflammation resolving process that can induce reparative macrophage phenotypes upon phagocytosis by the macrophages [22-24]. However, the capacity of macrophages to clear dead neutrophils is impaired by myelin phagocytosis $[26,27]$, which may lead to the release of cytotoxic neutrophil contents into the spinal cord parenchyma. Neutrophil apoptosis has been shown to be accelerated by the engagement of $\beta_{2}$ integrins, including CD11b/ CD18 (Mac-1) and CD11d/CD18, while in the presence of pro-inflammatory cytokines including TNF- $\alpha$ [52]. Syk mediates intracellular signaling downstream of Mac-1 and CD11d/CD18 [53], though the role of Syk in neutrophil cell death has not been determined. We found similarly upregulated levels of CD11b on infiltrated neutrophils in both genotypes, however, $\mathrm{Syk}^{\mathrm{f} / \mathrm{f}} \mathrm{MRP} 8-\mathrm{Cre}$ mice showed reduced cell death relative to $\mathrm{Syk}^{\mathrm{f} / \mathrm{f}}$ mice. These findings suggest that Syk signaling may participate in integrin-mediated acceleration of neutrophil cell death in the injured spinal cord, which could directly impact neurons and glia in the spinal cord or indirectly influence other inflammatory processes such as macrophage polarization. Abrogated neutrophil apoptosis may also contribute to the benefit observed with $\mathrm{CD} 11 \mathrm{~d} / \mathrm{CD} 18$ blocking antibody treatment after SCI [8]. 


\section{Conclusions}

Collectively, our results provide the first extensive characterization of neutrophil functional responses in the acutely injured cord and establish Syk signaling as a critical mediator of long-term neurologic deficits after SCI. We demonstrate, to the best of our knowledge, the first evidence of NET formation in murine SCI. This work represents a key step in understanding how neutrophils respond to the inflammatory environment of the injured spinal cord and identifies neutrophil cytokine expression and cell death as potential pathogenic events in the acutely injured spinal cord. Future studies are needed to determine how Syk signaling in neutrophils, as well as neutrophil cell death and cytokine expression, contribute to neurologic deficits independent of long-term tissue sparing.

\section{Abbreviations}

BMS: Basso Mouse Scale; Syk: Spleen tyrosine kinase; SCl: Spinal cord injury; G-MFI: Geometric mean fluorescent intensity; NETs: Neutrophil extracellular traps; ROS: Reactive oxygen species; RNS: Reactive nitrogen species; ITAMs: Immunoreceptor tyrosine activation motifs; NDS: Normal donkey serum; TBS: Tris-buffered saline.

\section{Acknowledgements}

Behavioral data were obtained with support from the UCSF Neurobehavioral Core for Rehabilitation Research. Use of the Texas A\&M Microscopy and Imaging Center is acknowledged.

\section{Authors' contributions}

$\mathrm{DM}, \mathrm{CA}$, and $\mathrm{CL}$ contributed to conception and design of the study. DM, YH, SM, MP, MK, SR, and FJ contributed to acquisition, analysis, and interpretation of data. DM drafted and CA and CL substantively revised the manuscript. All authors have approved the submitted version.

\section{Funding}

This work was funded by the NINDS F32NS096883 and R01NS122961, TIRR Foundation, and NIAID R01Al113272.

\section{Availability of data and materials}

The datasets used and/or analysed during the current study are available from the corresponding author on reasonable request.

\section{Declarations}

Ethics approval and consent to participate

All studies were performed in accordance with protocols approved by the Institutional Animal Care and Use Committee at the University of California, San Francisco.

\section{Consent for publication}

Not applicable.

\section{Competing interests}

The authors declare that they have no competing interests.

\section{Author details}

1 Department of Biology, Texas A\&M University, 301 Old Main Dr, ILSB 3128, College Station, TX 77843, USA. ${ }^{2}$ Texas A\&M Institute for Neuroscience, Texas A\&M University, College Station, TX 77843, USA. ${ }^{3}$ Department of Laboratory Medicine and Immunology Program, University of California, San Francisco, CA 94143, USA.
Received: 16 June 2021 Accepted: 14 December 2021

Published online: 24 December 2021

\section{References}

1. Ley K, Hoffman HM, Kubes P, Cassatella MA, Zychlinsky A, Hedrick CC, et al. Neutrophils: new insights and open questions. Sci Immunol. 2018;3(30):eaat4579.

2. Beck KD, Nguyen HX, Galvan MD, Salazar DL, Woodruff TM, Anderson AJ. Quantitative analysis of cellular inflammation after traumatic spinal cord injury: evidence for a multiphasic inflammatory response in the acute to chronic environment. Brain. 2010;133(Pt 2):433-47.

3. Stirling DP, Yong VW. Dynamics of the inflammatory response after murine spinal cord injury revealed by flow cytometry. J Neurosci Res. 2008;86(9):1944-58.

4. Brennan FH, Jogia T, Gillespie ER, Blomster LV, Li XX, Nowlan B, et al. Complement receptor $\mathrm{C} 3 \mathrm{aR} 1$ controls neutrophil mobilization following spinal cord injury through physiological antagonism of CXCR2. JCI Insight. 2019:4(9):e98254.

5. Jogia T, Lubstorf T, Jacobson E, Scriven E, Atresh S, Nguyen QH, et al. Prognostic value of early leukocyte fluctuations for recovery from traumatic spinal cord injury. Clin Transl Med. 2021;11(1):e272.

6. Zhao JL, Lai ST, Du ZY, Xu J, Sun YR, Yuan Q, et al. Circulating neutrophilto-lymphocyte ratio at admission predicts the long-term outcome in acute traumatic cervical spinal cord injury patients. BMC Musculoskelet Disord. 2020;21(1):548.

7. Saiwai H, Ohkawa Y, Yamada H, Kumamaru H, Harada A, Okano H, et al. The LTB4-BLT1 axis mediates neutrophil infiltration and secondary injury in experimental spinal cord injury. Am J Pathol. 2010;176(5):2352-66.

8. Gris D, Marsh DR, Oatway MA, Chen Y, Hamilton EF, Dekaban GA, et al. Transient blockade of the CD11d/CD18 integrin reduces secondary damage after spinal cord injury, improving sensory, autonomic, and motor function. J Neurosci. 2004;24(16):4043-51.

9. Taoka Y, Okajima K, Uchiba M, Murakami K, Kushimoto S, Johno M, et al. Role of neutrophils in spinal cord injury in the rat. Neuroscience. 1997:79(4):1177-82

10. Fleming JC, Bao F, Chen Y, Hamilton EF, Relton JK, Weaver LC. Alpha4 beta1 integrin blockade after spinal cord injury decreases damage and improves neurological function. Exp Neurol. 2008;214(2):147-59.

11. Lee SM, Rosen S, Weinstein P, van Rooijen N, Noble-Haeusslein LJ. Prevention of both neutrophil and monocyte recruitment promotes recovery after spinal cord injury. J Neurotrauma. 2011;28(9):1893-907.

12. Nguyen HX, Hooshmand MJ, Saiwai H, Maddox J, Salehi A, Lakatos A, et al. Systemic neutrophil depletion modulates the nigration and fate of transplanted human neural stem cells to rescue functional repair. J Neurosci. 2017;37(38):9269-87.

13. Saiwai H, Kumamaru H, Ohkawa Y, Kubota K, Kobayakawa K, Yamada H, et al. Ly6C+ Ly6G - Myeloid-derived suppressor cells play a critical role in the resolution of acute inflammation and the subsequent tissue repair process after spinal cord injury. J Neurochem. 2013;125(1):74-88.

14. Stirling DP, Liu S, Kubes P, Yong VW. Depletion of Ly6G/Gr-1 leukocytes after spinal cord injury in mice alters wound healing and worsens neurological outcome. J Neurosci. 2009;29(3):753-64.

15. Mayadas TN, Cullere X, Lowell CA. The multifaceted functions of neutrophils. Annu Rev Pathol. 2014;9:181-218.

16. Bentwood BJ, Henson PM. The sequential release of granule constitutents from human neutrophils. J Immunol. 1980;124(2):855-62.

17. Borregaard N, Cowland JB. Granules of the human neutrophilic polymorphonuclear leukocyte. Blood. 1997;89(10):3503-21.

18. Brinkmann V, Reichard U, Goosmann C, Fauler B, Uhlemann Y, Weiss DS, et al. Neutrophil extracellular traps kill bacteria. Science. 2004;303(5663):1532-5.

19. Lacy P. Mechanisms of degranulation in neutrophils. Allergy Asthma Clin Immunol. 2006;2(3):98-108.

20. Evans TJ, Buttery LD, Carpenter A, Springall DR, Polak JM, Cohen J. Cytokine-treated human neutrophils contain inducible nitric oxide synthase that produces nitration of ingested bacteria. Proc Natl Acad Sci USA. 1996;93(18):9553-8. 
21. Vethanayagam RR, Almyroudis NG, Grimm MJ, Lewandowski DC, Pham CT, Blackwell TS, et al. Role of NADPH oxidase versus neutrophil proteases in antimicrobial host defense. PLOS ONE. 2011;6(12):e28149.

22. Bosurgi L, Cao YG, Cabeza-Cabrerizo M, Tucci A, Hughes LD, Kong Y, et al. Macrophage function in tissue repair and remodeling requires IL-4 or IL-13 with apoptotic cells. Science. 2017;356(6342):1072-6.

23. Filardy AA, Pires DR, Nunes MP, Takiya CM, Freire-de-Lima CG, RibeiroGomes FL, et al. Proinflammatory clearance of apoptotic neutrophils induces an IL-12(low)IL-10(high) regulatory phenotype in macrophages. J Immunol. 2010;185(4):2044-50.

24. Marwick JA, Mills R, Kay O, Michail K, Stephen J, Rossi AG, et al. Neutrophils induce macrophage anti-inflammatory reprogramming by suppressing NF-kappaB activation. Cell Death Dis. 2018;9(6):665.

25. Poon IK, Lucas CD, Rossi AG, Ravichandran KS. Apoptotic cell clearance: basic biology and therapeutic potential. Nat Rev Immunol. 2014;14(3):166-80.

26. Wang $X$, Cao K, Sun $X$, Chen $Y$, Duan Z, Sun L, et al. Macrophages in spinal cord injury: phenotypic and functional change from exposure to myelin debris. Glia. 2015;63(4):635-51.

27. Guo L, Rolfe AJ, Wang X, Tai W, Cheng Z, Cao K, et al. Rescuing macrophage normal function in spinal cord injury with embryonic stem cell conditioned media. Mol Brain. 2016;9(1):48.

28. Tecchio C, Micheletti A, Cassatella MA. Neutrophil-derived cytokines: facts beyond expression. Front Immunol. 2014;5:508.

29. Mantovani A, Cassatella MA, Costantini C, Jaillon S. Neutrophils in the activation and regulation of innate and adaptive immunity. Nat Rev Immunol. 2011;11(8):519-31.

30. Scapini P, Lapinet-Vera JA, Gasperini S, Calzetti F, Bazzoni F, Cassatella MA. The neutrophil as a cellular source of chemokines. Immunol Rev. 2000;177:195-203.

31. Wright HL, Thomas HB, Moots RJ, Edwards SW. RNA-seq reveals activation of both common and cytokine-specific pathways following neutrophil priming. PLoS ONE. 2013;8(3):e58598.

32. Futosi K, Fodor S, Mocsai A. Reprint of Neutrophil cell surface receptors and their intracellular signal transduction pathways. Int Immunopharmacol. 2013;17(4):1185-97.

33. Lowell CA. Src-family and Syk kinases in activating and inhibitory pathways in innate immune cells: signaling cross talk. Cold Spring Harb Perspect Biol. 2011;3(3):a002352

34. van Rees DJ, Szilagyi K, Kuijpers TW, Matlung HL, van den Berg TK. Immunoreceptors on neutrophils. Semin Immunol. 2016;28(2):94-108.

35. Zarbock A, Ley K. Protein tyrosine kinases in neutrophil activation and recruitment. Arch Biochem Biophys. 2011;510(2):112-9.

36. Kiefer F, Brumell J, Al-Alawi N, Latour S, Cheng A, Veillette A, et al. The Syk protein tyrosine kinase is essential for Fcgamma receptor signaling in macrophages and neutrophils. Mol Cell Biol. 1998;18(7):4209-20.

37. Mocsai A, Abram CL, Jakus Z, Hu Y, Lanier LL, Lowell CA. Integrin signaling in neutrophils and macrophages uses adaptors containing immunoreceptor tyrosine-based activation motifs. Nat Immunol. 2006;7(12):1326-33.

38. Van Ziffle JA, Lowell CA. Neutrophil-specific deletion of Syk kinase results in reduced host defense to bacterial infection. Blood. 2009;114(23):4871-82

39. Negoro PE, Xu S, Dagher Z, Hopke A, Reedy JL, Feldman MB, et al. Spleen tyrosine kinase is a critical regulator of neutrophil responses to Candida species. MBio. 2020;11(3):1-8.

40. Elliott ER, Van Ziffle JA, Scapini P, Sullivan BM, Locksley RM, Lowell CA. Deletion of Syk in neutrophils prevents immune complex arthritis. J Immunol. 2011;187(8):4319-30.

41. Hirahashi J, Mekala D, Van Ziffle J, Xiao L, Saffaripour S, Wagner DD, et al. Mac-1 signaling via Src-family and Syk kinases results in elastase-dependent thrombohemorrhagic vasculopathy. Immunity. 2006;25(2):271-83.

42. Nemeth T, Virtic O, Sitaru C, Mocsai A. The Syk tyrosine kinase is required for skin inflammation in an in vivo mouse model of epidermolysis bullosa acquisita. J Invest Dermatol. 2017;137(10):2131-9.

43. Ozaki N, Suzuki S, Ishida M, Harada Y, Tanaka K, Sato Y, et al. Syk-dependent signaling pathways in neutrophils and macrophages are indispensable in the pathogenesis of anti-collagen antibody-induced arthritis. Int Immunol. 2012;24(9):539-50.
44. Nemeth T, Futosi K, Szilveszter K, Vilinovszki O, Kiss-Papai L, Mocsai A. Lineage-specific analysis of Syk function in autoantibody-induced arthritis. Front Immunol. 2018;9:555.

45. Salehi S, Wang X, Juvet S, Scott JA, Chow CW. Syk regulates neutrophilic airway hyper-responsiveness in a chronic mouse model of allergic airways inflammation. PLoS ONE. 2017;12(1):e0163614.

46. McCreedy DA, Lee S, Sontag CJ, Weinstein P, Olivas AD, Martinez AF, et al. Early targeting of L-selectin on leukocytes promotes recovery after spinal cord injury. Implicating novel mechanisms of pathogenesis. Eneuro. 2018;5:4.

47. Basso DM, Beattie MS, Bresnahan JC. Graded histological and locomotor outcomes after spinal cord contusion using the NYU weight-drop device versus transection. Exp Neurol. 1996;139(2):244-56.

48. Basso DM, Fisher LC, Anderson AJ, Jakeman LB, McTigue DM, Popovich PG. Basso Mouse Scale for locomotion detects differences in recovery after spinal cord injury in five common mouse strains. J Neurotrauma. 2006;23(5):635-59.

49. Kahn J, Ingraham RH, Shirley F, Migaki Gl, Kishimoto TK. Membrane proximal cleavage of L-selectin: identification of the cleavage site and a 6-kD transmembrane peptide fragment of L-selectin. J Cell Biol. 1994;125(2):461-70

50. Kishimoto TK, Jutila MA, Berg EL, Butcher EC. Neutrophil Mac-1 and MEL14 adhesion proteins inversely regulated by chemotactic factors. Science. 1989;245(4923):1238-41.

51. Winkler EA, Sengillo JD, Sagare AP, Zhao Z, Ma Q, Zuniga E, et al. Bloodspinal cord barrier disruption contributes to early motor-neuron degeneration in ALS-model mice. Proc Natl Acad Sci USA. 2014;111(11):E1035-42.

52. Mayadas TN, Cullere X. Neutrophil beta2 integrins: moderators of life or death decisions. Trends Immunol. 2005;26(7):388-95.

53. Mocsai A, Zhou M, Meng F, Tybulewicz VL, Lowell CA. Syk is required for integrin signaling in neutrophils. Immunity. 2002;16(4):547-58.

54. Sauerbeck AD, Laws JL, Bandaru VV, Popovich PG, Haughey NJ, McTigue DM. Spinal cord injury causes chronic liver pathology in rats. J Neurotrauma. 2015;32(3):159-69.

55. Ballesteros I, Rubio-Ponce A, Genua M, Lusito E, Kwok I, Fernandez-Calvo $\mathrm{G}$, et al. Co-option of neutrophil fates by tissue environments. Cell. 2020;183(5):1282-97.

56. Ghasemlou N, Bouhy D, Yang J, Lopez-Vales R, Haber M, Thuraisingam $T$, et al. Beneficial effects of secretory leukocyte protease inhibitor after spinal cord injury. Brain. 2010;133(Pt 1):126-38.

57. Gerber YN, Saint-Martin GP, Bringuier CM, Bartolami S, Goze-Bac C, Noristani HN, et al. CSF1R inhibition reduces microglia proliferation, promotes tissue preservation and improves motor recovery after spinal cord injury. Front Cell Neurosci. 2018;12:368.

58. Guan Z, Kuhn JA, Wang X, Colquitt B, Solorzano C, Vaman S, et al. Injured sensory neuron-derived CSF1 induces microglial proliferation and DAP12-dependent pain. Nat Neurosci. 2016;19(1):94-101.

\section{Publisher's Note}

Springer Nature remains neutral with regard to jurisdictional claims in published maps and institutional affiliations.

Ready to submit your research? Choose BMC and benefit from:

- fast, convenient online submission

- thorough peer review by experienced researchers in your field

- rapid publication on acceptance

- support for research data, including large and complex data types

- gold Open Access which fosters wider collaboration and increased citations

- maximum visibility for your research: over 100M website views per year

At BMC, research is always in progress.

Learn more biomedcentral.com/submissions 